\title{
Analysis and Design of Full-Bridge Class-DE Inverter at Fixed Duty Cycle
}

\author{
Luca Albertoni, Francesco Grasso, Jacopo Matteucci, \\ Maria C. Piccirilli, Alberto Reatti \\ DINFO-University of Florence \\ Via Santa Marta 3 I-50139 Florence \\ \{luca.albertoni, jacopo.matteucci\}@stud.unifi.it \\ \{francesco.grasso, mariacristina.piccirilli, alberto.reatti\}@unifi.it
}

\author{
Agasthya Ayachit, Marian K. Kazimierczuk \\ Department of Electrical Engineering \\ Wright State University \\ 3640 Colonel Glenn Hwy., \\ Dayton, OH, 45435, USA
}

\{ayachit.2, marian.kazimierczuk\}@wright.edu

\begin{abstract}
This paper presents the following for a full-bridge Class-DE resonant inverter operating at a fixed duty ratio: (a) steady-state analysis using first-harmonic approximation and (b) derivation of closed-form expressions for the currents, voltages, and powers. The conversion from a series-parallel resonant network to a series resonant network is presented. Imposing the zero-voltage and zero-derivative switching conditions, the expression for a shunt capacitance across the MOSFETs in the inverter bridge is derived. The closed-form expressions to calculate the values of the resonant components are presented. A practical design of a Class-DE resonant inverter supplied by a dc input voltage of $230 \mathrm{~V}$, delivering an output power of $920 \mathrm{~W}$, and operating at a switching frequency of $100 \mathrm{kHz}$ is considered and its design methodology is included. Theoretical results are validated by Saber simulations.
\end{abstract}

\section{INTRODUCTION}

Class-DE inverters are a family of power electronic topologies for energy conversion, which are duly characterized by high efficiency [1]-[4]. An interesting feature of the ClassDE topology is their ability to achieve both zero-voltage switching (ZVS) and zero-derivative switching (ZDS) conditions, thereby yielding high power-conversion efficiency, especially at high switching frequencies. This makes them suitable for applications such as RF power supplies and RF power amplifiers [3], induction heating [4], on-chip converters [5], [6], wireless-power transfer systems [7], [8], [20], etc. The Class-DE inverter shares a few features of Class-E as well as Class-D inverters making them an ideal choice for high-power applications [8], [12]-[19].

Several works in the literature related to the Class-DE inverter have focused on the following areas: steady-state analysis with half-bridge switching network at a fixed and any duty ratio [1], [5], [9], [12] and design of inverter with with linear and nonlinear shunt MOSFET capacitances [2], [14], [16], [17]. However, a detailed steady-state analysis of a full-bridge Class-DE inverter at any duty cycle has not been reported. Therefore, this paper aims at the following objectives: (a) develop a theoretical framework for the steady-state characteristics of the full-bridge Class-DE inverter operating at any duty ratio using the fundamental harmonic approximation, (b) derive the expressions for the shunt capacitances and the components in the resonant circuit, (c) develop a methodology

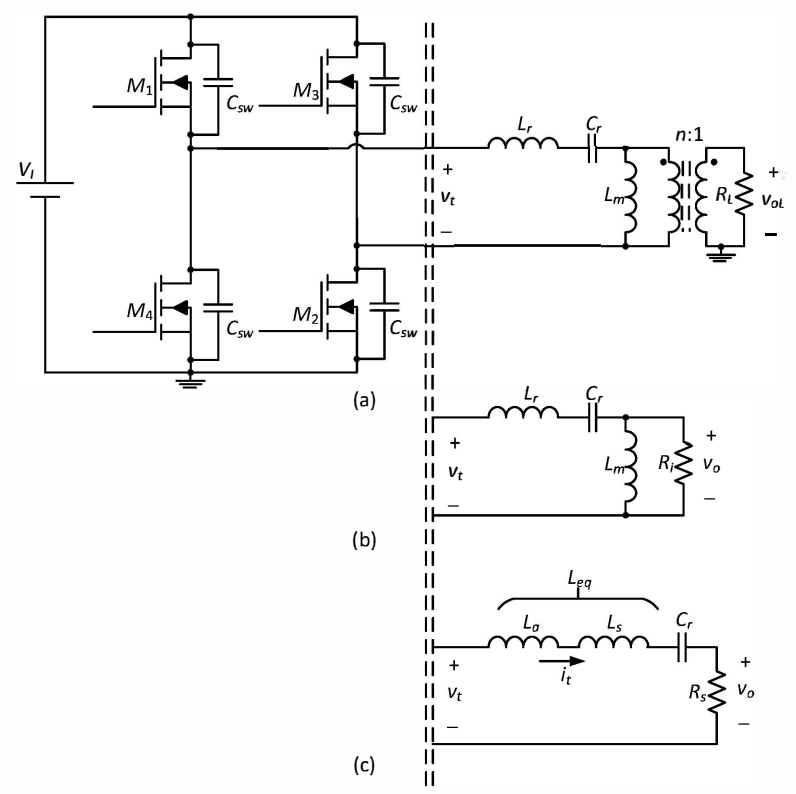

Fig. 1. Circuits of the full-bridge class-DE resonant inverter. (a) Series-parallel resonant circuit with transformer. (b) Series-parallel resonant circuit with load resistance on primary side. (c) Series resonant circuit.

to transform a series-parallel topology of the inverter to its series resonant form and vice versa, and (d) analyze its voltage transfer function, input impedance, and efficiency. A high-power, high-frequency full-bridge Class-DE inverter is designed. Saber circuit simulations are provided to validate the correctness of the theoretically analysis. The switchingnetwork waveforms demonstrate ZVS operation, while an efficiency of $97 \%$ is achieved.

\section{Full-BRIDGE ClAss-DE DC-AC INVERTER ANALYSIS}

\section{A. Circuit Description}

Fig. 1(a) shows the circuit of the full-bridge Class-DE series resonant inverter with a transformer. The circuit constitutes four MOSFETs each of them with a shunt capacitor $C_{s w}$, which are used to minimize the switching losses of these devices. The inverter is supplied by a DC voltage $V_{I}$ and 
feeds a resonant tank circuit with a square-wave voltage. The switching frequency is $f_{s}$. The anti-parallel diodes provides a path for the current to flow from the source to the drain during the instants, when the switches are OFF. The pulse-width of the gate signal has a duty cycle $D$. Each MOSFET remains ON for an interval $D T$, where $T=1 / f_{s}$ is the switching time period. The gate signals to the switches $M_{1}$ and $M_{4}$ are $180^{\circ}$ out of phase with those provided to the switches $M_{2}$ and $M_{3}$. The duty cycle chosen for each switch is lower than 0.5 , thereby providing a finite dead time between the switching instants. The shunt capacitors in parallel with the MOSFETs are used to shape the voltage across the MOSFETs in order to achieve ZVS condition under certain operating conditions.

In Fig. 1(a), the series resonant circuit consists of a resonant capacitor $C_{r}$ and a resonant inductor $L_{a}$, while $L_{m}$ represents the magnetizing inductance of the transformer. Fig. 1(b) illustrates the use of reflection principle to transfer the load resistance $R_{L}$ to the primary side forming the series-parallel resonant network. Fig. 1(c) shows the conversion of the seriesparallel network comprising of $L_{r}-C_{r}-L_{m}-R_{i}$ to a series resonant network comprising of $L_{a}-C_{r}-L_{s}-R_{s}$. Due to a high quality factor of these components, only the first harmonic of the tank voltage and current is delivered to the load.

\section{B. Circuit Analysis}

Analysis of a series resonant network is by far easier than the series-parallel topology. Therefore, a comprehensive analysis of the inverter is first performed on the series resonant circuit. Then, using appropriate transformation principles, the series topology shown in Fig. 1(a) is converted to seriesparallel topology shown in Fig. 1(b). The first harmonic approximation is used to determine the inverter currents and voltages [12]. The subsequent analysis of the inverter is based on the following assumptions:

1) The converter operates in steady state.

2) The current through the resonant tank is purely sinusoidal.

3) The circuit components are assumed to be ideal and all the parasitic components are neglected.

4) The DC supply voltage and the amplitude of the output voltage are constant.

5) Only the fundamental component is responsible for delivering the rated power to the load resistance.

The steady-state expressions and design equations consider a duty cycle of $D=0.4$. Therefore, each MOSFET is ON for $4 \pi / 5$. A symmetrical dead time of $t_{d}=\pi / 5$ is chosen at the beginning of each turn-ON event. The current through the resonant tank is

$$
i_{\text {tan }}=I_{m} \sin (\omega t+\phi),
$$

where $I_{m}$ is the amplitude of the sinusoidal current waveform, $\omega=2 \pi f_{s}$ is the angular operating frequency with $0<\omega t \leq$ $2 \pi$ and $\phi$ is the phase shift between the tank current and the tank voltage. Each switching period is divided into four time intervals as shown in Table I. Fig. 2 shows the key current and
TABLE I

Time Interval And STATE OF THE MOSFETS at $D=0.4$.

\begin{tabular}{|c|c|c|}
\hline Interval & Time & State \\
\hline$t_{A}=\left[t_{1}, t_{2}\right]$ & $\pi / 10 \leq \omega t<9 \pi / 10$ & $M_{1,2}$ on, $M_{3,4}$ off \\
$t_{B}=\left[t_{2}, t_{3}\right]$ & $9 \pi / 10 \leq \omega t<11 \pi / 10$ & $M_{1,2,3,4}$ off \\
$t_{C}=\left[t_{3}, t_{4}\right]$ & $11 \pi / 10 \leq \omega t<19 \pi / 10$ & $M_{1,2}$ on, $M_{3,4}$ off \\
$t_{D}=\left[t_{4}, t_{1}\right]$ & $19 \pi / 10 \leq \omega t<21 \pi / 10$ & $M_{1,2,3,4}$ off \\
\hline
\end{tabular}

voltage waveforms during indicating the state of the inverter during each sub-interval. During time interval $t_{A}, M_{1}$ and $M_{2}$ are $\mathrm{ON}$ to give

$$
\begin{gathered}
v_{D S 1}=v_{D S 2}=0 \\
v_{D S 3}=v_{D S 4}=V_{I} .
\end{gathered}
$$

Since the voltage across the shunt capacitances are constant, their currents are equal to zero. During the interval $t_{B}$, the capacitors $C_{s w 1}$ and $C_{s w 2}$ begin to charge, while the capacitors $C_{s w 3}$ and $C_{s w 4}$ begin to discharge. Applying the KVL and KCL to the nodes and the meshes of the circuit, we obtain the following equations.

$$
\begin{gathered}
V_{I}=v_{D S 1}+v_{D S 4}=v_{D S 3}+v_{D S 2}, \\
i_{\text {tan }}=i_{C S W 1}-i_{C S W 4}=i_{C S W 2}-i_{C S W 3} .
\end{gathered}
$$

Combination of (3) and (4) and their derivative results in

$$
\left\{\begin{aligned}
i_{\text {tan }} & =\omega C_{s w 1} \frac{d v_{D S 1}}{d(\omega t)}-\omega C_{s w 4} \frac{d v_{D S 4}}{d(\omega t)} \\
i_{\text {tan }} & =\omega C_{s w 2} \frac{d v_{D S 2}}{d(\omega t)}-\omega C_{s w 3} \frac{d v_{D S 3}}{d(\omega t)}
\end{aligned}\right.
$$

where

$$
\left\{\begin{array}{c}
\frac{d v_{D S 1}}{d(\omega t)}=-\frac{d v_{D S 4}}{d(\omega t)}=\frac{I_{m}}{\omega\left(C_{s w 1}+C_{s w 4}\right)} \sin (\omega t+\phi) \\
\frac{d v_{D S 2}}{d(\omega t)}=-\frac{d v_{D S 3}}{d(\omega t)}=\frac{I_{m}}{\omega\left(C_{s w 2}+C_{s w 3}\right)} \sin (\omega t+\phi .)
\end{array}\right.
$$

Imposing zero-derivative switching (ZDS) for the voltages across the MOSFETs, during turn $\mathrm{ON}$ at $\omega t=\pi / 10$, we get

$$
\sin \left(\frac{\pi}{10}+\phi\right)=0
$$

to yield

$$
\phi=-\frac{\pi}{10} .
$$

Thus, solving for capacitor currents provide

$$
\left\{\begin{array}{c}
i_{C s w 1}=\frac{C_{s w 1}}{\left(C_{s w 1}+C_{s w 4}\right)} I_{m} \sin \left(\omega t-\frac{\pi}{10}\right) \\
i_{C s w 2}=\frac{C_{s w 2}}{\left(C_{s w 2}+C_{s w 3}\right)} I_{m} \sin \left(\omega t-\frac{\pi}{10}\right) \\
i_{C s w 3}=-\frac{C_{s w 3}}{\left(C_{s w 2}+C_{s w 3}\right)} I_{m} \sin \left(\omega t-\frac{\pi}{10}\right) \\
i_{C s w 4}=-\frac{C_{s w 4}}{\left(C_{s w 1}+C_{s w 4}\right)} I_{m} \sin \left(\omega t-\frac{\pi}{10}\right)
\end{array}\right.
$$

and solving for the switch voltages yields

$$
\left\{\begin{array}{c}
v_{D S 1}=\frac{I_{m}}{\omega\left(C_{s w 1}+C_{s w 4}\right)}\left[-\cos \left(\omega t+\frac{\pi}{10}\right)+\cos \left(\frac{\pi}{5}\right)\right] \\
v_{D S 2}=\frac{I_{m}}{\omega\left(C_{s w 2}+C_{s w 3}\right)}\left[-\cos \left(\omega t+\frac{\pi}{10}\right)+\cos \left(\frac{\pi}{5}\right)\right] \\
v_{D S 3}=\frac{I_{m}}{\omega\left(C_{s w 2}+C_{s w 3}\right)}\left[-\cos \left(\omega t+\frac{\pi}{10}\right)+\cos \left(\frac{\pi}{5}\right)\right]+V_{I} \\
v_{D S 4}=\frac{I_{m}}{\omega\left(C_{s w 1}+C_{s w 4}\right)}\left[-\cos \left(\omega t+\frac{\pi}{10}\right)+\cos \left(\frac{\pi}{5}\right)\right]+V_{I} .
\end{array}\right.
$$

Imposing the zero-voltage switching (ZVS) condition to $v_{D S 3}$ 


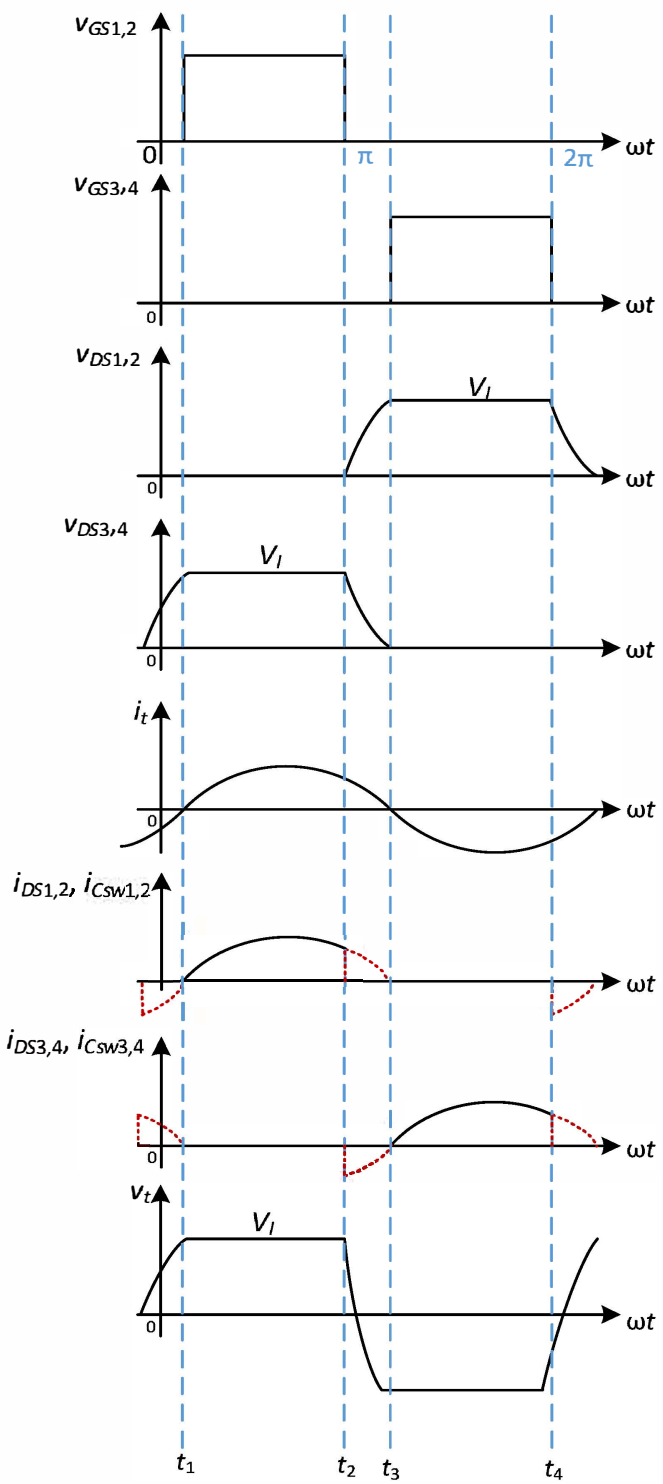

Fig. 2. Waveforms of switch currents, switch voltages, capacitor current, resonant current, and the resonant tank voltage.

and $v_{D S 4}$ at the end of time interval $t_{B}$ at $\omega t=11 \pi / 10$, we get

$$
C_{s w 1}+C_{s w 4}=C_{s w 2}+C_{s w 3},
$$

to give

$$
I_{m}=\frac{C_{s w 1}+C_{s w 4}}{1-\cos \frac{\pi}{5}} \omega V_{I}=5.236\left(C_{s w 1}+C_{s w 4}\right) \omega V_{I}
$$

During time interval $t_{C}, M_{3}$ and $M_{4}$ are ON. Therefore,

$$
\begin{gathered}
v_{D S 1}=v_{D S 2}=V_{I} \\
v_{D S 3}=v_{D S 4}=0 .
\end{gathered}
$$

Finally, during the interval $t_{D}$, the circuit configuration is dual with respect to that achieved during time interval $t_{B}$, where the currents and voltage are displaced exactly by $180^{\circ}$. The current and voltage expressions are

$$
\begin{gathered}
i_{C s w 1}=\frac{C_{s w 1}}{\left(C_{s w 1}+C_{s w 4}\right)} I_{m} \sin \left(\omega t-\frac{\pi}{10}\right) \\
i_{C s w 2}=\frac{C_{s w 2}}{\left(C_{s w 2}+C_{s w 3}\right)} I_{m} \sin \left(\omega t-\frac{\pi}{10}\right) \\
i_{C s w 3}=-\frac{C_{s w 3}}{\left(C_{s w 2}+C_{s w 3}\right)} I_{m} \sin \left(\omega t-\frac{\pi}{10}\right) \\
i_{C s w 4}=-\frac{C_{s w 4}}{\left(C_{s w 1}+C_{s w 4}\right)} I_{m} \sin \left(\omega t-\frac{\pi}{10}\right) .
\end{gathered}
$$

and

$$
\left\{\begin{array}{c}
v_{D S 1}=\frac{I_{m}}{\omega\left(C_{s w 1}+C_{s w 4}\right)}\left[\cos \left(\omega t+\frac{\pi}{10}\right)-\cos \left(\frac{\pi}{5}\right)\right] \\
v_{D S 2}=\frac{I_{m}}{\omega\left(C_{s w 2}+C_{s w 3}\right)}\left[+\cos \left(\omega t+\frac{\pi}{10}\right)-\cos \left(\frac{\pi}{5}\right)\right] \\
v_{D S 3}=\frac{I_{m}}{\omega\left(C_{s w_{2}}+C_{s w 3}\right)}\left[-\cos \left(\omega t+\frac{\pi}{10}\right)+\cos \left(\frac{\pi}{5}\right)\right]+V_{I} \\
v_{D S 4}=\frac{I_{m}}{\omega\left(C_{s w 1}+C_{s w 4}\right)}\left[-\cos \left(\omega t+\frac{\pi}{10}\right)+\cos \left(\frac{\pi}{5}\right)\right]+V_{I} .
\end{array}\right.
$$

\section{ClOSED-FORM EXPRESSIONS}

\section{A. Currents, Voltages, and Powers}

The average dc input current can be determined as follows. During $t_{A}$ between $\frac{\pi}{10}<\omega t \leq \frac{9 \pi}{10}, i_{\text {tan }}=i_{D S 1}$. Similarly, during $t_{C}$ between $\frac{11 \pi}{10}<\omega t \leq \frac{19 \pi}{10}, i_{\text {tan }}=i_{D S 2}$. By considering the overall tank current using (5), we obtain the average dc input current as

$$
I_{I}=\frac{1}{2 \pi} \int_{0}^{2 \pi} i_{t a n} d(\omega t)=\frac{I_{m}}{2 \pi} .
$$

Similarly, the tank voltage applied to the resonant circuit is

$$
v_{t a n}=v_{D S 3}-v_{D S 1} \text {. }
$$

A Fourier series expansion of $v_{\text {tan }}$ given in (17) yields

$$
v_{\text {tan }}=\frac{1}{2} a_{0}+\sum_{n=1}^{\infty} a_{n} \cos (n \omega t-\phi)+b_{n} \sin (n \omega t-\phi) \text {. }
$$

In (18), $a_{0}=0$, since $v_{\text {tan }}$ has odd symmetry. From Fig. 1 , at resonance, the voltage drops across $C_{r}$ and $L_{a}$ are equal and out of phase by $180^{\circ}$ canceling out each other. Thus, for the fundamental component $(n=1)$ and the applied tank voltage becomes

$v_{t a n}=v_{L s}+v_{o}=V_{L m} \cos \left(\omega t-\frac{\pi}{10}\right)+V_{m} \sin \left(\omega t-\frac{\pi}{10}\right)$.

The Fourier coefficients are $a_{n}=V_{L m}$ and $b_{n}=V_{m}$, whose expressions are determined as follows. From the principle of Fourier series, the amplitude of the voltage across the inductance $L_{s}$ is defined as

$$
\begin{aligned}
V_{L m} & =\frac{1}{\pi} \int_{0}^{2 \pi}\left(v_{D S 3}-v_{D S 1}\right) \cos \left(\omega t+\frac{9 \pi}{10}\right) d(\omega t) \\
& =V_{I} \frac{\sin \frac{8 \pi}{5}+\frac{2 \pi}{5}}{\pi\left(1+\cos \frac{4 \pi}{5}\right)}=6.76 V_{I} .
\end{aligned}
$$

Similarly, the output voltage amplitude is

$$
V_{m}=\frac{1}{\pi} \int_{0}^{2 \pi}\left(v_{D S 3}-v_{D S 1}\right) \sin \left(\omega t+\frac{9 \pi}{10}\right) d(\omega t)=\frac{4 V_{I}}{\pi} .
$$


Applying Ohm's Law, $V_{L m}=I_{m} \omega L_{s}$ and $V_{m}=I_{m} R_{s}$. Therefore, the ratio $V_{L m} / V_{m}$ is

$$
\frac{V_{L m}}{V_{m}}=\frac{\omega L_{s}}{R_{s}}=\frac{6.76 V_{I}}{\frac{4 V_{I}}{\pi}}=5.31 .
$$

Furthermore, the output power is given by

$$
P_{O}=\frac{V_{m}^{2}}{2 R_{s}}=\frac{\left[\frac{2}{\pi} V_{I}\left(1-\cos \frac{4}{5} \pi\right)\right]^{2}}{2 R_{s}},
$$

from which

$$
R_{s}=\frac{V_{m}^{2}}{2 P_{O}}
$$

The input power of the inverter is $P_{I}=V_{I} I_{m}$. Using (12)

$$
\begin{aligned}
P_{I} & =V_{I} I_{m}=\frac{\omega V_{I}^{2}}{\pi} \frac{1-\cos \frac{4}{5} \pi}{1+\cos \frac{4}{5} \pi}\left(C_{s w 1}+C_{s w 4}\right) \\
& =3.01 \omega V_{I}^{2}\left(C_{s w 1}+C_{s w 4}\right) .
\end{aligned}
$$

\section{B. Circuit Components}

Assuming the inverter efficiency as unity, the $P_{O}=P_{I}$. If the shunt capacitances are equal to $C_{s w}$, then combining (11) and (25) provides

$$
C_{s w}=\frac{\pi P_{O}}{\omega V_{I}^{2}} \frac{1+\cos \frac{4}{5} \pi}{1-\cos \frac{4}{5} \pi}=0.33 \frac{P_{O}}{\omega V_{I}^{2}} .
$$

The quality factor of the series resonant tank responsible to provide a pure sinusoidal current waveform is

$$
Q_{s}=\frac{\omega_{s}\left(L_{a}+L_{s}\right)}{R_{s}}=\frac{\omega_{s} L}{R_{s}} .
$$

Modifying (27), the inductance $L_{a}$ is

$$
L_{a}=L-L_{s}=\left(Q_{s}-\frac{V_{L m}}{V_{m}}\right) \frac{R_{s}}{\omega_{s}},
$$

where $V_{L m} / V_{m}$ is as given in (22). The components $L_{a}$ and $C_{r}$ are tuned to resonate at the switching frequency $f_{s}$. Therefore,

$$
\omega_{s}=\frac{1}{\sqrt{L_{a} C_{r}}}=2 \pi f_{s} .
$$

The resonant capacitance is expressed as

$$
C_{r}=\frac{1}{\omega_{s}^{2} L_{a}}=\frac{1}{\omega_{s} R_{s}\left(Q_{s}-\frac{V_{L m}}{V_{m}}\right)} .
$$

Since $L=L_{a}+L_{s}$, the the resonant frequency between $C_{r}$ and $L$ is

$$
\omega_{p}=\frac{1}{\sqrt{L C_{r}}} .
$$

The inductance $L_{s}$ in the series form can be converted into $L_{m}$ in parallel to the load inductance. In the version of the inverter as shown in Fig. 1(a), $L_{m}$ represents the magnetizing inductance of transformer. Let the loaded quality factor be

$$
q_{l}=\frac{\omega_{s} L_{s}}{R_{s}} .
$$

Converting the series $L_{s}-R_{s}$ combination to a parallel $L_{m}-$ $R_{p}$ network has been described in [12]. Thus, the parallel inductance $L_{m}$ and the resistance $R_{p}$ are

$$
\begin{aligned}
& L_{m}=L_{s}\left(1+\frac{1}{q_{l}^{2}}\right) . \\
& R_{p}=R_{s}\left(1+q_{\text {load }}^{2}\right) .
\end{aligned}
$$

\section{Voltage Transfer Function And EFFICIENCY}

\section{A. Voltage Transfer Function}

From the waveforms in Fig. 2, the tank voltage at the input of the resonant tank

$v_{\text {tan }}=\frac{-2 I_{m}}{\omega\left(C_{s w 2}+C_{s w 3}\right)}\left[\sin \left(\omega t+\frac{4 \pi}{10}\right)+\cos \left(\frac{\pi}{5}\right)\right]+V_{I}$,

for the intervals $0 \leq \omega t<\frac{3}{10} \pi$ and $\frac{17}{10} \pi \leq \omega t<\frac{23}{10} \pi$. During the intervals $\frac{3}{10} \pi \leq \omega t<\frac{7}{10} \pi$ and $\frac{13}{10} \pi \leq \omega t<\frac{17}{10} \pi$, the value of the tank voltage is $V_{I}$. Similarly, during $\frac{7}{10} \pi \leq \omega t<\frac{13}{10} \pi$ $v_{\text {tan }}=\frac{2 I_{m}}{\omega\left(C_{s w 2}+C_{s w 3}\right)}\left[\sin \left(\omega t+\frac{4 \pi}{10}\right)-\cos \left(\frac{\pi}{5}\right)\right]+V_{I}$

Combining these voltages, the fundamental component is

$$
\begin{aligned}
v_{t a n 1} & =V_{t a n 1 m} \sin (\omega t-\theta) \\
& =V_{L m} \cos \left(\omega t-\frac{3}{5} \pi\right)+V_{m} \sin \left(\omega t-\frac{3}{5} \pi\right),
\end{aligned}
$$

where

$$
V_{\tan 1 m}=\sqrt{V_{L m}^{2}+V_{m}^{2}}, \text { and } \theta=\phi+\psi .
$$

In (38), $\theta$ is the phase of the fundamental tank voltage, $\phi=-\pi / 10$ as obtained in (8), and $\psi$ is the phase shift introduced by the resonant tank. The rms value of the fundamental tank voltage is $V_{\tan 1 \mathrm{rms}}=V_{\tan 1 \mathrm{~m}} / \sqrt{2}$. The voltage transfer function of the full-bridge Class-DE inverter is

$$
M_{V S}=\frac{V_{t a n 1 r m s}}{V_{I}}
$$

and the voltage transfer function of the resonant circuit is

$$
M_{V R}=\frac{v_{o}}{V_{t a n 1 r m s}}=\left|M_{v r}\right| e^{j \alpha} .
$$

Thus, the overall voltage transfer function of the inverter is

$$
\begin{aligned}
M_{V I} & =M_{V S} M_{V R}=\frac{v_{o}}{V_{I}} \\
& =\frac{\sqrt{V_{L m}^{2}+V_{m}^{2}}}{\sqrt{2} V_{I}}\left|M_{v r}\right| e^{j \alpha} .
\end{aligned}
$$

In (41), the magnitude $\left|M_{v r}\right|$ and phase $\alpha$ are

$$
\left|M_{V R}\right|=\frac{1}{\sqrt{(1+A)^{2}\left[1-f_{n}^{2}\right]^{2}+\frac{1}{Q_{L}^{2}}\left(\frac{1}{f_{n}} \frac{A}{A+1}-f_{n}\right)^{4}}}
$$




$$
\alpha=\tan ^{-1}\left[\frac{1}{Q_{L}} \frac{\left(\frac{1}{f_{n}} \frac{A}{A+1}-f_{n}\right)^{2}}{(1+A)\left(1-f_{n}^{2}\right)}\right],
$$

respectively. The ratio of the resonant to magnetizing inductance is

$$
A=\frac{L}{L_{m}}
$$

The ratio of the series-parallel resonant frequency to the switching frequency is the normalized frequency given by

$$
f_{n}=\frac{f_{p}}{f_{s}} .
$$

The quality factor of the series-parallel tank is

$$
Q_{p}=\omega_{p} C_{r} R_{p}=\frac{R_{p}}{\omega_{p}\left(L_{r}+L_{m}\right)} .
$$

\section{B. Input Impedance}

The characteristic impedance of the resonant circuit is

$$
Z_{o}=\sqrt{\frac{L_{r}+L_{m}}{C}}
$$

The input impedance of the series-parallel tank is

$$
Z_{i}=R_{p} \frac{(1+A)\left(1-f_{n}^{2}\right)+j \frac{1}{Q_{\boldsymbol{P}}}\left(\frac{1}{f_{n}} \frac{A}{1+A}-f_{n}\right)}{1-j Q_{P} f_{n}(1+A)} .
$$

Thus, the ratio of the magnitude of the input impedance to the characteristic impedance is

$$
\frac{\left|Z_{i}\right|}{Z_{O}}=Q_{p} \sqrt{\frac{(1+A)^{2}\left(1-f_{n}^{2}\right)^{2}+\frac{1}{Q_{P}^{2}}\left(\frac{1}{f_{n}} \frac{A}{1+A}-f_{n}\right)^{2}}{1+\left[Q_{P} f_{n}(1+A)\right]^{2}}} .
$$

Using (38) and (49), the amplitude of the current through the resonant tank is

$$
I_{\tan 1 m}=\frac{V_{\tan 1 m}}{\left|Z_{i}\right|}=\frac{V_{L m}^{2}+V_{m}^{2}}{\left|Z_{i}\right|} .
$$

\section{Efficiency}

The overall power loss in the resonant circuit is [12]

$$
\begin{aligned}
P_{\text {loss }} & =P_{C r}+P_{L r}+P_{D S}+P_{C s w} \\
& =\left(r_{C r}+r_{L r}\right) I_{\text {tan } 1 r m s}^{2}+4 r_{D S} I_{D S r m s}^{2} \\
& +8 r_{C s w} I_{C \text { swrms }}^{2},
\end{aligned}
$$

where the rms value of the tank current is

$$
I_{t a n 1 r m s}=\frac{I_{t a n 1 m}}{\sqrt{2}}
$$

the rms value of the MOSFET current is

$$
I_{D S r m s}=\frac{I_{\tan 1 m}}{2} \sqrt{\frac{1}{2 \pi}\left[\frac{8}{5} \pi-\sin \left(\frac{8}{5} \pi\right)\right]}=0.487 I_{\tan 1 \mathrm{~m}},
$$

the rms value of the current through the shunt capacitance is

$$
I_{C s w r m s}=\frac{I_{\tan 1 m}}{2} \sqrt{\frac{1}{8 \pi}\left[\frac{2}{5} \pi+\sin \left(\frac{8}{5} \pi\right)\right]}=0.055 I_{\tan 1 \mathrm{~m}}
$$

The rms value of the current through the load resistance is

$$
I_{\text {Rprms }}=\frac{1}{R_{p}} \sqrt{\frac{V_{L m}^{2}+V_{m}^{2}}{2}}\left|M_{v r}\right| .
$$

Therefore, the inverter output power is

$$
P_{R p}=I_{R p r m s}^{2} R_{p}=\frac{V_{L m}^{2}+V_{m}^{2}}{2 R_{p}} M_{v r}^{2} .
$$

Finally, the inverter efficiency can be determined as

$$
\eta_{I}=\frac{1}{1+\frac{P_{R p_{-}}}{P_{l \bullet s s}}}
$$

\section{Class-DE Inverter Simulation Results}

\section{A. Design Example}

An inverter with design specification as follows is designed: DC input voltage $V_{I}=230 \mathrm{~V}$, output power $P_{O}=921 \mathrm{~W}$, and switching frequency $f_{s}=100 \mathrm{kHz}$. A duty cycle $D=0.4$ is considered. The dead time is $t_{d}=0.1$. Using (14), the values of the shunt capacitances are $C_{s w}=4.25 \mathrm{nF}$. Let us assume the quality factor $Q_{s}=10$. From (12), $R_{s}=37.2 \Omega$, from (15), $L=592 \mu \mathrm{H}$, from (16) $L_{a}=565.65 \mu \mathrm{H}$ to yield $L_{s}=26.35 \mu \mathrm{H}$, and from (17), $C_{r}=4.48 \mathrm{nF}$. For the seriesparallel resonant circuit we have $L_{m}=166.2 \mu \mathrm{H}$ and $R_{p}=$ 44.1 $\Omega$. The parasitic resistances of the MOSFET are $r_{D S}=$ $15 \mathrm{~m} \Omega$, shunt and resonant capacitor $r_{C s w}=r_{C r}=0.656 \Omega$, and resonant inductor $r_{L}=0.110 \Omega$. According to (31), the inverter voltage transfer function is $M_{V I}=0.89$. Thus, the amplitude of the output voltage of the series-parallel network is $V_{m}=204 \mathrm{~V}$. Furthermore, the overall inverter power loss was calculated as $P_{\text {loss }}=23.3 \mathrm{~W}$. The inverter efficiency calculated using (57) was $\eta_{I}=97.6 \%$.

\section{B. Simulation Results}

The inverter designed in the previous section and shown in Fig. 1 was simulated on SABER circuit simulator. Fig. 3 shows the waveforms of the current and voltage waveforms of MOSFETs $S_{1}$ illustrating the ZVS operation. A small portion of the switch current flows through the anti-parallel diode, causing its drain-source voltage to be equal to zero. Fig. 4 shows the waveforms of the tank current $i_{t a n}$, tank voltage $v_{t a n}$, output voltage $v_{o}$, and output power $p_{R p}$. The rms value of the output current and voltage were $4.98 \mathrm{~A}$ and $185 \mathrm{~V}$, respectively. The measured rms output power was $921 \mathrm{~W}$, the measured input power was $958 \mathrm{~W}$. The measured overall efficiency was $96 \%$ confirming the theoretical analysis. 


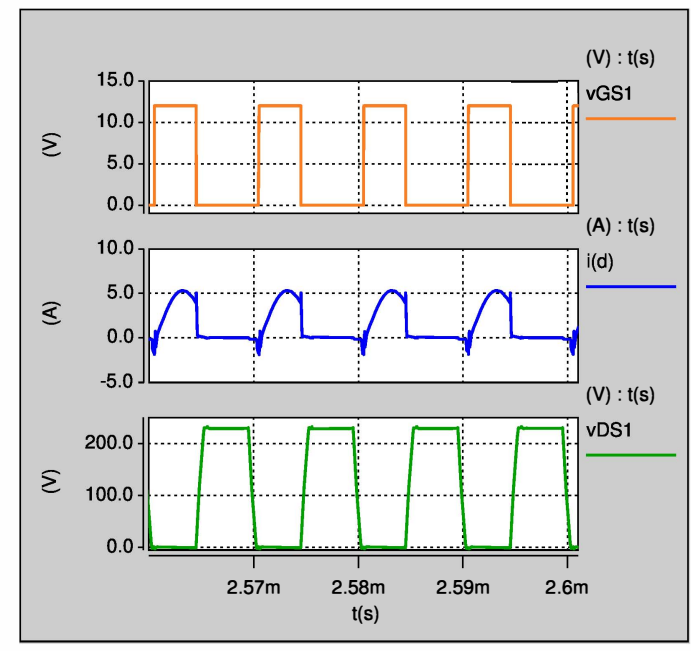

Fig. 3. Simulated waveforms of the gate-to-source voltage $v_{G S 1}$, drain-tosource voltage $v_{D S 1}$, and drain current $i_{D 1}$ of the MOSFET $S_{1}$ showing ZVS operation.

\section{CONCLUSIONS}

This paper has presented the following for a full-bridge Class-DE resonant inverter operating at a fixed duty ratio: (a) steady-state analysis using first-harmonic approximation and (b) derivation of closed-form expressions for the currents, voltages, and powers. The design procedure has been formulated for a series resonant tank circuit. Using transformation principles, the series resonant network has been transformed into a series-parallel resonant topology in order to accommodate the magnetizing inductance of the transformer. The expressions to calculate the values of resonant circuit component and the wave-shaping shunt capacitances of the inverter-bridge have been derived. The voltage transfer function and the input impedance of the series-parallel topology have been analyzed. The expression for the overall efficiency of the inverter has been derived. Simulations have been performed on an inverter operating at a supply voltage of $230 \mathrm{~V}$, output power of 920 $\mathrm{W}$, and a switching frequency of $100 \mathrm{kHz}$. The simulation results show the validity of the theoretical predicted analysis.

\section{REFERENCES}

[1] H. Sekiya, X. Wei, T. Nagashima, and M. K. Kazimierczuk, "Steady state analysis and design of class-DE inverter at any duty ratio," IEEE Trans. Power Electron., vol. 30, no. 7, pp. 3685-3694, July 2015.

[2] H. Sekiya, N. Sagawa, and M. K. Kazimierczuk, "Analysis of class DE amplifier with nonlinear shunt capacitances at any grading coefficient for high Q and 25\% duty ratio," IEEE Trans. Power Electron., vol. 25, no. 4, pp. 924-932, Apr. 2010.

[3] M. Amjad, Z. Salam, M. Facta, and S. Mekhilef, "Analysis and implementation of transformerless LCL resonant power supply for ozone generation," IEEE Trans. Power Electron., vol. 28, no. 2, pp. 650-660, Feb. 2013.

[4] H. Sarnago, O. Lucia, A. Mediano, and J. M. Burdio, "Class-D/DE dualmode-operation resonant converter for improved-efficiency domestic induction heating system," IEEE Trans. Power Electron., vol. 28, no. 3, pp. 1274-1285, Mar. 2013.

[5] T. Suetsugu and M. K. Kazimierczuk, "Integration of class DE inverter for on-chip power supplies," Proc. IEEE Intl. Symp. Circ. Syst., Island of Kos, Greece, May 2006, pp. 3133-3136.

[6] T. Suetsugu and M. K. Kazimierczuk, "Integration of class DE dcdc converter for on-chip power supplies," Proc. IEEE Power Electron. Specialist Conf., Jeju, South Korea, Jun. 2006, pp. 1-5.

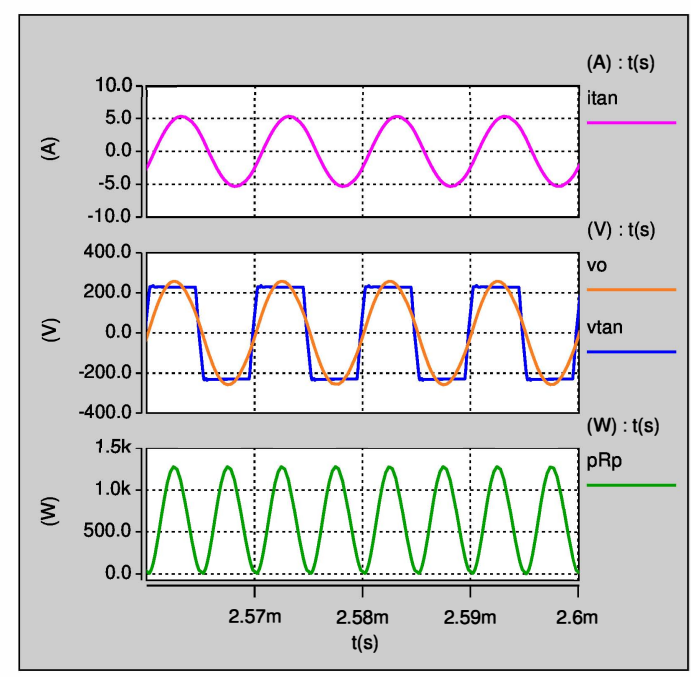

Fig. 4. Simulated waveforms of the tank current $i_{t a n}$, tank voltage $v_{t a n}$, output voltage $v_{o}$, and output power $p_{R p}$.

[7] D. Murthy-Bellur, A. Bauer, W. Kerin, and M. K. Kazimierczuk, "Inverter using loosely coupled inductors for wireless power transfer," Proc. IEEE Intl. Midwest Symp. Circ. Syst., Boise, ID, USA, Aug. 2012, pp.1164-1167.

[8] K. Inoue, T. Nagashima, X. Wei, and H. Sekiya, "Design of highefficiency inductive-coupled wireless power transfer system with classDE transmitter and class-E rectifier,' Proc. IEEE Industrial Electronics Society, Vienna, Austria, Nov. 2013, pp. 613-618.

[9] M. K. Kazimierczuk, N. Thirunarayan, and S. Wang, "Analysis of series parallel resonant converter," IEEE Trans. Aerosp. and Electron. Syst., vol. 29 , no. 1 , pp. 88-99, 1993.

[10] J. Modzelewski, "Optimum and sub-optimum operation of highfrequency Class-D zero-voltage-switching tuned power amplifier," Bull. Polish Acad. Sci., Tech. Sci., vol. 46, no. 4, pp. 458-473, Apr. 1998.

[11] M. K. Kazimierczuk and J. Jozwik, "Resonant dc/dc converter with class E inverter and class-E rectifier," IEEE Trans. Ind. Electron., vol. 36, no. 4, pp. 468-478, Apr. 1989.

[12] M. K. Kazimierczuk, RF Power Amplifiers, 2nd Ed., John Wiley Sons, Chichester, UK, 2014.

[13] M. K. Kazimierczuk and D. Czarkowski, Resonant Power Converters, 2nd Ed., John Wiley Sons, Hoboken, NJ, 2012.

[14] M. Hayati, A. Lotfi, M. K. Kazimierczuk, and H. Sekiya, "Analysis and design of class-E power amplifier with MOSFET parasitic linear and nonlinear capacitances at any duty ratio," IEEE Trans. Power Electron., vol. 28, no. 11, pp. 5222-5232, Nov. 2013.

[15] A. Mediano and P. Molina, "Frequency limitation of a high-efficiency class E tuned RF power amplifier due to a shunt capacitance," Proc. IEEE MTT-S Intl. Microwave. Symp. Dig., Anaheim, CA, USA, Jun. 1999, pp. 13-19.

[16] A. Mediano, P. Molina, and J. Navarro, "Class E RF/microwave power amplifier: Linear Equivalent of transistors nonlinear output capacitance, normalized design and maximum operating frequency versus output capacitance," Proc. IEEE MTT-S Int. Microw. Symp. Dig., Boston, MA, USA, Jun., 2000, pp. 783-786.

[17] X. Wei, H. Sekiya, S. Kuroiwa, T. Suetsugu, and M. K. Kazimierczuk, "Design of class-E amplifier with MOSFET linear gate-to-drain and nonlinear drain-to-source capacitances," IEEE Trans. Circuits Syst.-I, vol. 58, no. 10 , pp. $2556-2565$, Oct. 2011.

[18] T. Suetsugu and M. K. Kazimierczuk, "Maximum operating frequency of class-E amplifier at any duty ratio," IEEE Trans. Circ. Syst.-II, vol. 55, no. 8, pp. 768-770, Aug. 2008.

[19] M. K. Kazimierczuk and W. Szaraniec, "Class D zero-voltage switching inverter with only one shunt capacitor," IEE Proceedings, Part B, Electric Power Appl., vol. 139, pp. 449-456, Sept. 1999.

[20] A. Ayachit, D. K. Saini, T. Suetsugu, and M. K. Kazimierczuk, "Threecoil wireless power transfer system using Class-E2 resonant dc-dc converter," Proc. IEEE Intl. Telecommunications Energy Conf., INTELEC, Yokohama, Japan, October 2015, pp. 1116-1119. 